\title{
Pancreatic perfusion and arterial-transit-time quantification using pseudocontinuous arterial spin labeling at $3 \mathrm{~T}$
}

\section{Citation}

Taso, M., A. Guidon, L. Zhao, K. Mortele, and D. Alsop. 2018. Pancreatic perfusion and arterialtransit-time quantification using pseudocontinuous arterial spin labeling at 3T. Magnetic Resonance in Medicine.

\section{Permanent link}

http://nrs.harvard.edu/urn-3:HUL.InstRepos:38035040

\section{Terms of Use}

This article was downloaded from Harvard University's DASH repository, and is made available under the terms and conditions applicable to Open Access Policy Articles, as set forth at http:// nrs.harvard.edu/urn-3:HUL.InstRepos:dash.current.terms-of-use\#OAP

\section{Share Your Story}

The Harvard community has made this article openly available. Please share how this access benefits you. Submit a story. 


\title{
Pancreatic perfusion and arterial-transit-time (ATT) quantification using pseudo-continuous Arterial Spin Labeling (pCASL) at 3T
}

\author{
Manuel Taso ${ }^{1,2, *}$, Arnaud Guidon ${ }^{3}$, Li Zhao ${ }^{1,2}$, Koenraad J. Mortele ${ }^{1,2}$, David C. Alsop ${ }^{1,2}$ \\ ${ }^{1}$ Division of MRI Research, Department of Radiology, Beth Israel Deaconess Medical \\ Center, Boston, $M A$ \\ ${ }^{2}$ Harvard Medical School, Boston, MA \\ ${ }^{3}$ Global MR Applications and Workflow, GE Healthcare, Boston, MA
}

\section{Corresponding author}

Manuel Taso, Ph.D.

Beth Israel Deaconess Medical Center

Division of MRI Research - Dept. of Radiology

330, Brookline Avenue - Ansin 244

Boston, MA 02215

mtaso@bidmc.harvard.edu

+1 (617) 667-0290

Number of words: 2974

Number of references: 35

Number of tables: 1

Number of figures: 4

Key-words: Arterial Spin Labeling (ASL), Pancreas, Perfusion, PCASL, Arterial Transit Time (ATT) 


\section{Abstract}

Purpose: To demonstrate the feasibility of non-invasively measuring pancreatic perfusion using pseudo-continuous Arterial Spin Labeling (ASL) and to derive quantitative bloodflow and transit-time measurements in healthy volunteers.

Methods: A pseudo-continuous ASL sequence with background suppression and a singleslice single-shot Fast-Spin-Echo readout was acquired at 3 Tesla in 10 subjects with a single standard post-labeling delay (PLD) of 1.5s and in 4 additional subjects with 4 PLD from 0.7 to $2 \mathrm{~s}$. An imaging synchronized breathing approach was used to minimize motion artifacts during the 3 minutes of acquisition. Scan-rescan reproducibility was assessed in 3 volunteers with single-delay ASL. Quantitative blood-flow and arterial transit-time (ATT) were derived and the impact of ATT correction was studied using either subject-specific ATT in the second group or an average ATT derived from the group with multi-delay ASL for subjects with single-delay ASL.

Results: Successful ASL acquisitions were performed in all volunteers. An average pancreatic blood-flow of $201 \pm 40 \mathrm{~mL} / 100 \mathrm{~g} / \mathrm{min}$ was measured in the single-delay group using an assumed ATT of $750 \mathrm{~ms}$. Average ATT measured in the multi-delay group was $1029 \pm 89 \mathrm{~ms}$. Using the longer, measured ATT reduced the measured flow to $162 \pm 12$ and $168 \pm 28 \mathrm{~mL} / 100 \mathrm{~g} / \mathrm{min}$ with subject-specific or average ATT correction, respectively. ASL signal heterogeneities were observed at shorter PLD, potentially linked to its complex vascular supply and islet distribution.

Conclusions: ASL enables reliable measurement of pancreatic perfusion in healthy volunteers. It presents a valuable alternative to contrast-enhanced methods and may be useful for diagnosis and characterization of several inflammatory, metabolic and neoplastic diseases affecting the pancreas. 


\section{Introduction}

Non-invasive functional pancreas imaging presents high clinical relevance to diagnose, grade and monitor multiple neoplastic, inflammatory or metabolic diseases. For instance, pancreas perfusion could be a valuable marker of endocrine dysfunction in diabetes (1), assess necrosis and fibrosis in acute/chronic pancreatitis, help discrimination between benign and malignant pancreatic masses (2) or different disease phenotypes (3) and assess treatment response in pancreatic cancer, for example by monitoring anti-angiogenic effects of targeted therapies (4).

Arterial Spin Labeling (ASL) $(5,6)$ is an interesting alternative to more widely used contrast-enhanced modalities $\left({ }^{15} \mathrm{O}-\mathrm{PET}\right.$, CT perfusion or DCE-MRI $)(1,7,8)$ due to several advantages, such as repeatability due to the absence of contrast agent injection or ionizing radiation, applicability in patients with poor renal function, and simplified straightforward quantification compared to DCE-MRI. However, while clinically relevant, abdominal ASL has not been as successfully translated to clinical applications as in neuroimaging. Some challenges of abdominal ASL include intrinsic low sensitivity, respiratory and physiological (cardiac, peristalsis) motion leading to signal instability and errors when subtracting control and label experiments, as well as magnetic $\left(\mathrm{B}_{0}\right)$ and radiofrequency field $\left(B_{1}\right)$ inhomogeneities affecting labeling efficiency (9). These must all be addressed for robust and reproducible body imaging, especially at 3 Tesla (T). Finally, although straightforward, ASL-based blood-flow quantification relies on prior knowledge or measurement of $\mathrm{T}_{1}$ relaxation and arterial transit-time (ATT) between the labeling location and tissue of interest.

Among the reports using ASL in abdominal organs, applications in the pancreas are rare in the literature due to the above-mentioned challenges but also relatively short longitudinal relaxation time $T_{1}$ (10) causing rapid signal decay. A few previous studies $(11,12)$ have reported the use of ASL with pulsed-labeling methods such as FAIR at $1.5 \mathrm{~T}$ in healthy subjects, mainly demonstrating the feasibility of the technique and calculating regional 
blood-flow modulation following secretin injection (13). While successful pioneering studies, image quality assessment is not possible from the published work nor did they measure ATT, an essential determinant of both blood flow quantification and optimal labeling timing.

In the current work, we sought to investigate the performance of pancreatic perfusion measurement in healthy volunteers at $3 \mathrm{~T}$ with ASL, offering higher signal-to-noise ratio (SNR) than 1.5T. This work also differs from previous studies by using a pseudocontinuous ASL (pCASL) labeling method (14), known for having an increased sensitivity compared to FAIR strategies and by using very strong background suppression (15) to minimize the effects of bulk and physiological motion. After first demonstrating perfusion images in the pancreas and quantifying absolute blood-flow, we emphasized measuring ATT from multiple post-labeling delays (PLD) to assess its influence on blood-flow quantification. 


\section{Material and Methods}

\section{Study design}

Two different sub-studies are presented in the current work:

- \#1: assessing whether measuring pancreatic perfusion is feasible when single-delay pCASL is used at $3 \mathrm{~T}$

- \#2: quantifying ATT and determining its influence on blood-flow quantification using multi-delay ASL

\section{Cohort}

A total of 14 ( 7 men and 7 women, mean age $29 \pm 8$ y.o, range $22-41$ y.o) healthy volunteers were enrolled in this study. Written informed consent was obtained prior to scanning, and the study protocol was approved by the institutional Committee on Clinical Investigations. A first cohort of 10 volunteers underwent the single-delay ASL protocol and the remaining 4 volunteers underwent the multi-delay ASL protocol.

All scans were performed on a 3T scanner (Discovery MR750, GE Healthcare, Waukesha, WI), with body coil RF transmission and signal reception using a 32-channel body array coil.

\section{MR Imaging}

Three-plane localizers followed by axial and coronal breath-hold $\mathrm{T}_{2}$-weighted single-shot Fast-Spin-Echo (SSFSE) scans were acquired for pancreas localization and ASL slice positioning.

ASL was then acquired with a timed-breathing strategy, known to mitigate motion artifacts $(16,17)$ and the subjects were coached to hold their breath during the imaging readout (less than a second) and breathe during the remaining time before the next readout window. Perfusion images were acquired with a single-slice axial pCASL-SSFSE sequence with the following readout parameters: $\mathrm{TR} / \mathrm{TE}=6000 / 45 \mathrm{~ms}$, receiver bandwidth $=19.23 \mathrm{kHz}$, echo 
spacing $5.9 \mathrm{~ms}, 72$ echoes echo train $\left(1^{\text {st }} 155^{\circ}\right.$ refocusing pulse followed by a train of $130^{\circ}$ flip angles) no parallel imaging, FOV=30-32cm, slice thickness $10 \mathrm{~mm}, 128 \times 128$ matrix. The slice was positioned to cover as much pancreatic tissue as possible based on $\mathrm{T}_{2}-\mathrm{W}$ localizers.

Fourteen pairs of label/control data were acquired as well as three reference images (one pre-saturated PD-weighted and two inversion-recovery images with TI=190 and $1570 \mathrm{~ms}$ for $\mathrm{M}_{0}$ and $\mathrm{T}_{1}$ quantification purposes) in 3 minutes. In the volunteers with multi-delay ASL acquisitions, four successive ASL sequences were acquired with different PLDs for ATT quantification.

\section{Arterial Spin Labeling}

ASL was acquired using a background-suppressed unbalanced pCASL preparation (14) with the following parameters: Hann-shaped $500 \mu$ s pulses, repetition rate $1.18 \mathrm{~ms}$, peak RF amplitude $6.6 \mu \mathrm{T}$, average/maximum labeling gradient strength $0.5 / 3.5 \mathrm{mT} / \mathrm{m}$. The lower gradient strength than in many previous reports and recommendations (18) was employed due to recent findings of increased robustness towards pulsatile flow and $\mathrm{B}_{0}$ inhomogeneities (9). Labeling was applied with an average RF strength of $1.4 \mu \mathrm{T}$ for $1.5 \mathrm{~s}$, followed by a variable PLD of $0.7,1,1.5$ and $2 \mathrm{~s}$ for the multi-delay group and $1.5 \mathrm{~s}$ for the single-delay group. More detailed descriptions of the labeling can be found in earlier studies $(16,17)$. The labeling was performed axially, around $7-8 \mathrm{~cm}$ above the slice center to label descending blood in the aorta (Figure 1).

The BS was implemented and optimized for each PLD as previously described using a combination of inversion/saturation pulses $(16,19,20)$ leaving around $1-2 \%$ of the static tissue signal.

For qualitative comparison, we also acquired axial, single-slice pre-saturated FAIR perfusion images at the same location in 3 volunteers $(\mathrm{TI}=2.5 \mathrm{~s}, \tau=1 \mathrm{~s}$ (first saturation pulse), background suppression and inferior/superior saturation slabs) with an implementation previously described $(21,22)$. 


\section{Image reconstruction and processing}

\section{Image reconstruction}

Data were saved as raw signal intensities and an offline reconstruction was performed using MATLAB (R2015a, MathWorks, Natick, MA). Complex k-space subtraction of control and label data $\left(\mathrm{dM}=\mathrm{M}_{\text {control}}-\mathrm{M}_{\text {label }}\right)$ was performed followed by perfusion averaging for each PLD dataset. Then Half-Fourier homodyne reconstruction to a 256x256 matrix was performed (23) followed by phased coil combination.

$T_{1}$ and Arterial transit-time (ATT) estimation

For $\mathrm{T}_{1}$ quantification, a fitting from the two IR images normalized by the first saturated image to the following signal equation was performed as described previously (16):

$$
\frac{M_{\text {inv }}(T I)}{M_{\text {sat }}}=\frac{1+\exp \left(-\frac{t_{\text {sat }}}{T_{1}}\right)-2 \exp \left(-\frac{T I}{T_{1}}\right)}{1-\exp \left(-\frac{t_{s a t}}{T_{1}}\right)}
$$

For ATT quantification, the approach proposed by Dai et al. (24) was implemented as it provides a more computational-time efficient quantification of transit times. Briefly, it relies on the calculation of a signal-weighted delay defined as:

$$
W D=\left[\sum_{i=1}^{4} w_{i} \Delta M\left(\delta, w_{i}\right)\right] /\left[\sum_{i=1}^{4} \Delta M\left(\delta, w_{i}\right)\right]
$$

where $\mathrm{w}_{\mathrm{i}}(\mathrm{i}=1, . ., 4)$ is the PLD used in each scan, and $\Delta M\left(\delta, w_{i}\right)$ is the perfusion difference for a transit delay $\delta$ at the PLD wi. A range of WD was simulated for each $\mathrm{T}_{1}$ value found in each image pixel for transit delays from 0.7 to $2 \mathrm{~s}$ and a fixed blood $\mathrm{T}_{1}$ of $1.66 \mathrm{~s}$. Then, the ATT value for each pixel was obtained by numerically inverting the transit delay from the WD, as this latter is a monotonic function of the assumed transit delay. It was found that the 1s PLD presented a systematically lower signal than theoretically expected, inconsistent with the known perfusion signal kinetics. A search for the cause of attenuation at this PLD located a sequence implementation error in the BS pulse timing for this delay 
only that caused partial saturation of the image slice. Therefore, this PLD was excluded from the ATT estimation.

Non-ATT corrected and ATT corrected absolute blood flow quantification

An absolute pancreatic blood-flow (PBF) was calculated using a standard kinetic model $(25,26)$ for both studies:

$$
\mathrm{d} M=2 M_{t}^{0} \alpha T_{1, t} f e^{-\frac{\delta}{T_{1, b}}}\left(e^{-\max (\mathrm{w}-\delta, 0) / T_{1, t}}-e^{-\max (\tau+w-\delta, 0) / T_{1, t}}\right) / \lambda
$$

With $\mathrm{dM}$ the measured perfusion difference, $\mathrm{M}_{0, \mathrm{t}}$ the estimated fully-relaxed magnetization, $\tau=1.5 \mathrm{~s}$ the labeling time, $\mathrm{w}=1.5 \mathrm{~s}$ the PLD (for single-delay ASL), $\delta$ the arterial transit-time (estimated at $750 \mathrm{~ms}$ originally), $T_{1, b}=1.66 \mathrm{~s}$ and $T_{1, t}$ the blood and tissue longitudinal relaxation times (27), $\lambda=0.9$ the blood-tissue partition coefficient, $\alpha=0.6$ the labeling efficiency (assumed to be 0.8 for labeling and 0.75 for the BS inversions) (28). For Study 2 volunteers, with multi-delay ASL and quantified ATT, an additional bloodflow map was calculated by using the subject-specific ATT map estimated previously. Additionally, an ATT-corrected blood-flow was also calculated for the subjects scanned with single-delay ASL in Study 1 using an average pancreatic ATT measured from Study 2.

\section{Statistical analysis}

To assess PBF reproducibility, three volunteers from the $1^{\text {st }}$ group were scanned twice at least one week apart to calculate an average coefficient of variation, defined as the mean of the individual COVs:

$$
C O V_{P B F}=\frac{\sigma_{P B F}}{\mu_{P B F}}
$$

With $\sigma_{P B F}$ and $\mu_{P B F}$ the standard deviation (SD) and average of PBF measurements. Additionally, we calculated an intra-subject correlation coefficient defined as the Pearson correlation coefficient between the $1^{\text {st }}$ and $2^{\text {nd }}$ measurement. 
A relative SNR was calculated on the average perfusion difference for each subject by dividing the mean signal value of a region of interest (ROI) in the pancreas by the standard deviation of a ROI in the background noise. In addition, a relative SNR per unit of time (rSNRt) was calculated as the SNR normalized by the square root of the acquisition time (number of label/control pairs).

A ROI encompassing as much pancreatic tissue as could be reliably seen on the perfusion difference image was used for all quantification. In the $1^{\text {st }}$ study cohort, mean and in-ROI $\mathrm{SD}$ of $\mathrm{T}_{1}$, and pancreatic blood-flow were calculated. In the $2^{\text {nd }}$ study cohort, the ATT was quantified, as well as non-corrected and ATT-corrected pancreatic blood-flow. A paired two-tailed Student t-test was performed to assess any significant difference of blood-flow due to ATT correction.

Finally, the data for the $1^{\text {st }}$ study cohort were additionally corrected for ATT using an average ATT from the multi-delay cohort, and here again a paired two-tailed Student t-test was performed to assess any significant difference of blood-flow due to ATT correction. All statistics were performed using JMP Pro 13 (SAS, Cary, NC, USA), p-values $<0.05$ were considered statistically significant. 


\section{Results}

\section{Study 1: Pancreatic perfusion measurement feasibility and reproducibility}

A reliable perfusion signal could be observed in the pancreas in all volunteers (Figure 2). When comparing FAIR and pCASL perfusion-weighted images, significant vascular contamination can be observed on the FAIR images (yellow arrows) arising in major vessels. Reversely, the pCASL images show only tissue perfusion in the pancreas, kidneys and spleen, free from overwhelming vascular signal.

SNR and rSNRt measures (table 1) in the pancreas are sufficient, with an average SNR in the pancreas close to 10. Even in cases with some missed breathing instructions (Supplementary figure S1), the ASL image exhibits detectable perfusion in the pancreas. Nevertheless, residual strong vascular signal could be observed in major vessels in some cases (extreme left and middle-right images) leading to appreciable flow-artifacts in the phase-encoding direction.

Pancreatic blood-flow, quantified with an assumed ATT of 750ms and an averaged measured $T_{1}$ of $852 \pm 82 \mathrm{~ms}$ (in-line with previous literature reports) (10), was measured to be an average of $201 \pm 40 \mathrm{~mL} / 100 \mathrm{~g} / \mathrm{min}$, consistent with some of the previously reported values $(11,12)$. It is worth noting the elevated within-ROI standard deviation, representing on average $30 \%$ of the mean value, suggesting strong heterogeneity in pancreatic tissue perfusion. Also, some inter-subject variability can be seen in PBF measurement, estimated at $19 \%$.

Scan-rescan reproducibility yielded an inter-scan $\mathrm{COV}=14 \%$ and an intra-subject correlation of 0.79 .

\section{Study 2: ATT quantification and influence on blood-flow quantification and retrospective correction}


A clear post-labeling delay dependence of ASL difference signal within the pancreas was observed (figure 3). At longer PLDs, the ASL signal in the pancreas also appears more homogeneous compared to shorter delays (1s and below), in which we can observe some heterogeneity in the pancreatic perfusion signal, with an edge enhanced appearance. Particularly, the pancreatic tail seems to be presenting a higher perfusion signal at shorter delays.

ATT measurements (table 1) reveal a longer value than previously used in the preliminary quantification (1029 \pm 89 versus $750 \mathrm{~ms}$ ), hence implying an initial overestimation of pancreatic blood-flow. Similarly to PBF observations, it can be seen that the ATT presents an important in-ROI variability, around $26 \%$ of the average ATT value. As seen on figure 4, the pancreatic tail appears to have a shorter ATT compared to the rest of the pancreatic tissue. In contrast, the ATT inter-subject variation appears lower than PBF (8.6\%). Using measured subject-specific ATT values for quantification results in a lower PBF compared to naively quantified PBF with an assumed ATT of $750 \mathrm{~ms}$ (162 \pm 12 vs $191 \pm 15$ $\mathrm{mL} / 100 \mathrm{~g} / \mathrm{min}, \mathrm{p}=0.005)$. Interestingly, one can appreciate on fig. 4 the effect of ATT correction when looking at the heterogeneity of pancreatic blood-flow. The tail appears to present a higher blood-flow than the rest of the pancreas, something not consistent with a previous report (12).

Similar to the multi-delay results, blood-flow quantification using an average of the multidelay ATT led to a significantly lower estimated blood-flow, (201 vs $169 \mathrm{~mL} / 100 \mathrm{~g} / \mathrm{min}$ for the non-corrected vs corrected, $\mathrm{p}<0.0001)$. 


\section{Discussion}

We have shown that ASL at 3T using background-suppressed pseudo-continuous ASL and a timed-breathing approach can provide reliable measurements of pancreatic perfusion. Furthermore, we have quantified an average ATT and observed its influence on the accuracy of PBF quantification.

This work considerably strengthens the literature on ASL of pancreas. As previously mentioned, only a few reports of measurement of absolute pancreatic bloodflow using ASL could be found in the literature. While these earlier reports calculated PBF in regions of interest defined on anatomic images, they did not provide perfusion images, or presented perfusion images compromised by large vessel signal in close proximity $(29,30)$. Our study clearly demonstrated pancreatic perfusion signal, permitting an assessment of image quality, artifacts, and heterogeneity of flow, showing benefits of a localized pCASL labeling strategy in the abdomen, where residual vascular signal associated with a global labeling approach (e.g. FAIR) could compromise image quality and robustness as seen in some earlier reports and in the current work. The absolute PBF estimated in our study is nonetheless in-line with previous reports using MRI $(11,12)$ and $\left[{ }^{15} \mathrm{O}\right] \mathrm{H}_{2} 0$-PET (1), although substantial variability was found in existing literature. For instance, our results are in agreement with previous ASL work by Cox et al. (11) and DCE by Bali et al. (7), but lower by $30 \%$ than another ASL study by Schraml et al. (12), while remaining within the standard deviation of that study. In addition to a higher intraindividual correlation (0.79) than a previous study (13), we report a scan-rescan COV of $14 \%$. This latter could probably be improved by controlling systematically the scanning conditions (fasting, physical activity).

We observed visible heterogeneity in both PBF and ATT that was confirmed by quantification of within ROI variability. Several factors may contribute to these heterogeneities. First, unlike some other abdominal organs (e.g. kidneys), the pancreas is not solely supplied by one artery but by several arteries (branches of the SMA and splenic artery). This may contribute to a heterogeneity in blood-flow and ATT between the pancreatic head, body and tail, that has been previously highlighted $(11,12)$ and can be seen 
in the current work through potential edge enhancement effects at shorter delays. Additionally, signs of edge enhancement in early delay images were observed, suggesting initial arrival in vasculature near the outer surface of the pancreas that then gradually penetrates into the deeper tissue.

Furthermore, as pancreatic islets are known to receive up to $20 \%$ of the total pancreatic flow (31) while representing a small portion of its mass, it is possible that some blood-flow and ATT heterogeneities could be related to the islet distribution heterogeneity, considering that previous histological work have demonstrated that the islet concentration is higher in the body-tail region of the pancreas (32).

Our measured transit time to the pancreas, $1029 \pm 89 \mathrm{~ms}$, represents the first reported measurement attempt of ATT in the pancreas. Therefore, a longer PLD should be used to remove potential transit-delay weighting and intravascular signal contamination as observed in our data at short PLD (700ms), especially in major vessels (e.g. abdominal aorta) in the phase-encoding direction. Vessel-suppression strategies as previously described (24) could also be of assistance in limiting remaining intravascular signal. Future studies may also benefit from ATT measurement methods with higher SNR and time efficiency, including reduced resolution transit delay pre-scanning (24) or Hadamard timeencoded ASL (33). Still regarding ATT, the effect of refocusing pulses and gradients in sequences such as GRASE and FSE on vascular attenuation in ASL experiments has been recently emphasized (34). Our estimate of diffusion sensitivity at k-space center using the methods of Weigel et al. (35) is $b_{\text {eff }} \approx 2 \mathrm{~s} / \mathrm{mm}^{2}$. This modest diffusion sensitivity may attenuate ASL signal in larger vessels, especially at short PLDs where some labeled blood might still be in the vascular compartment, hence potentially overestimating ATT. Such attenuation may merit further study, especially when more complex, two compartment models for quantification are employed.

A limitation of the current work was the use of a single axial slice, precluding total pancreatic coverage. Since we could not systematically obtain a single axial slice covering pancreatic head/body/tail, one ROI covering the entire observable pancreas was drawn. Implementing multi-slice or 3D readouts would enable better study of regional variations. 
However, 2D multi-slice acquisitions are poorly compatible with background suppression and 3D readouts still present long acquisition times potentially leading to degraded image quality (17). Better spatial coverage of the pancreas is an important goal for future work.

The feasibility of obtaining high-quality perfusion data in the pancreas using ASL demonstrated here suggests numerous potential clinical applications, especially for the diagnosis or monitoring of inflammatory, metabolic or neoplastic pathologies affecting the pancreas. 


\section{Conclusion}

In conclusion, pancreatic perfusion measurement using pCASL at $3 \mathrm{~T}$ was proven to be feasible, with high-quality images and satisfactory SNR obtained in 3 minutes using a timed-breathing strategy. Thanks to multi-delay ASL acquisition, quantification of ATT was possible, resulting in an average measured ATT of $1029 \pm 89 \mathrm{~ms}$, suggesting that a longer post-labeling delay should be used than in prior studies. The average ATT-corrected blood-flow was measured to be $162 \pm 12 \mathrm{~mL} / 100 \mathrm{~g} / \mathrm{min}$ with subject-specific ATT correction and $169 \pm 27 \mathrm{~mL} / 100 \mathrm{~g} / \mathrm{min}$ with an average global ATT correction. Our technique and findings help establish a baseline for future research and clinical applications. 


\section{Acknowledgements}

The authors gratefully acknowledge Fotini Kourtelidis, Teresa Russell and Meaghan Fox for their support in conducting this study. 


\section{References}

1. Carlbom L, Espes D, Lubberink M, Eriksson O, Johansson L, Jansson L, Korsgren O, Ahlström H, Carlsson P-O. Pancreatic perfusion and subsequent response to glucose in healthy individuals and patients with type 1 diabetes. Diabetologia 2016;59:1968-1972. doi: $10.1007 / \mathrm{s} 00125-016-4016-2$.

2. Yadav AK, Sharma R, Kandasamy D, Pradhan RK, Garg PK, Bhalla AS, Gamanagatti S, Srivastava DN, Sahni P, Upadhyay AD. Perfusion CT - Can it resolve the pancreatic carcinoma versus mass forming chronic pancreatitis conundrum? Pancreatology 2016;16:979-987. doi: 10.1016/j.pan.2016.08.011.

3. Ma W, Li N, Zhao W, et al. Apparent Diffusion Coefficient and Dynamic ContrastEnhanced Magnetic Resonance Imaging in Pancreatic Cancer: Characteristics and Correlation With Histopathologic Parameters. J. Comput. Assist. Tomogr. 2016;40:709716. doi: 10.1097/RCT.0000000000000434.

4. Akisik MF, Sandrasegaran K, Bu G, Lin C, Hutchins GD, Chiorean EG. Pancreatic Cancer: Utility of Dynamic Contrast-enhanced MR Imaging in Assessment of Antiangiogenic Therapy. Radiology 2010;256:441-449. doi: 10.1148/radiol.10091733.

5. Detre JA, Zhang W, Roberts DA, Silva AC, Williams DS, Grandis DJ, Koretsky AP, Leigh JS. Tissue specific perfusion imaging using arterial spin labeling. NMR Biomed. $1994 ; 7: 75-82$.

6. Williams DS, Detre JA, Leigh JS, Koretsky AP. Magnetic resonance imaging of perfusion using spin inversion of arterial water. Proc. Natl. Acad. Sci. U. S. A. 1992;89:212-216.

7. Bali MA, Metens T, Denolin V, De Maertelaer V, Devière J, Matos C. Pancreatic perfusion: noninvasive quantitative assessment with dynamic contrast-enhanced $\mathrm{MR}$ imaging without and with secretin stimulation in healthy volunteers--initial results. Radiology 2008;247:115-121. doi: 10.1148/radiol.2471070685.

8. Tsushima Y, Miyazaki M, Taketomi-takahashi A, Endo K. Feasibility of Measuring Human Pancreatic Perfusion In Vivo Using Imaging Techniques. Pancreas 2011;40:747752. doi: 10.1097/MPA.0b013e318215ac22.

9. Zhao L, Vidorreta M, Soman S, Detre JA, Alsop DC. Improving the robustness of pseudo-continuous arterial spin labeling to off-resonance and pulsatile flow velocity. Magn. Reson. Med. 2016. doi: 10.1002/mrm.26513.

10. de Bazelaire CMJ, Duhamel GD, Rofsky NM, Alsop DC. MR imaging relaxation times of abdominal and pelvic tissues measured in vivo at 3.0 T: preliminary results. Radiology 2004;230:652-659. doi: 10.1148/radiol.2303021331. 
11. Cox EF, Smith JK, Chowdhury AH, Lobo DN, Francis ST, Simpson J. Temporal assessment of pancreatic blood flow and perfusion following secretin stimulation using noninvasive MRI. J. Magn. Reson. Imaging JMRI 2015;42:1233-1240. doi: 10.1002/jmri.24889.

12. Schraml C, Schwenzer NF, Martirosian P, Claussen CD, Schick F. Perfusion imaging of the pancreas using an arterial spin labeling technique. J. Magn. Reson. Imaging JMRI 2008;28:1459-1465. doi: 10.1002/jmri.21564.

13. Schawkat K, Ith M, Christe A, Kühn W, Chittazhathu Y, Bains L, Runge VM, Heverhagen JT. Dynamic non-invasive ASL perfusion imaging of a normal pancreas with secretin augmented MR imaging. Eur. Radiol. 2018:1-8. doi: 10.1007/s00330-017-52278.

14. Dai W, Garcia D, de Bazelaire C, Alsop DC. Continuous flow-driven inversion for arterial spin labeling using pulsed radio frequency and gradient fields. Magn. Reson. Med. 2008;60:1488-1497. doi: 10.1002/mrm.21790.

15. Ye FQ, Frank JA, Weinberger DR, McLaughlin AC. Noise reduction in 3D perfusion imaging by attenuating the static signal in arterial spin tagging (ASSIST). Magn. Reson. Med. 2000;44:92-100. doi: 10.1002/1522-2594(200007)44:1<92::AIDMRM14>3.0.CO;2-M.

16. Robson PM, Madhuranthakam AJ, Dai W, Pedrosa I, Rofsky NM, Alsop DC. Strategies for reducing respiratory motion artifacts in renal perfusion imaging with arterial spin labeling. Magn. Reson. Med. 2009;61:1374-1387. doi: 10.1002/mrm.21960.

17. Robson PM, Madhuranthakam AJ, Smith MP, Sun MRM, Dai W, Rofsky NM, Pedrosa I, Alsop DC. Volumetric Arterial Spin-labeled Perfusion Imaging of the Kidneys with a Three-dimensional Fast Spin Echo Acquisition. Acad. Radiol. 2016;23:144-154. doi: 10.1016/j.acra.2015.09.013.

18. Alsop DC, Detre JA, Golay X, et al. Recommended implementation of arterial spinlabeled perfusion MRI for clinical applications: A consensus of the ISMRM perfusion study group and the European consortium for ASL in dementia. Magn. Reson. Med. 2015;73:102-116. doi: 10.1002/mrm.25197.

19. Maleki N, Dai W, Alsop DC. Optimization of background suppression for arterial spin labeling perfusion imaging. Magma N. Y. N 2012;25:127-133. doi: 10.1007/s10334-0110286-3.

20. Mani S, Pauly J, Conolly S, Meyer C, Nishimura D. Background suppression with multiple inversion recovery nulling: applications to projective angiography. Magn. Reson. Med. 1997;37:898-905.

21. De Bazelaire C, Rofsky NM, Duhamel G, Michaelson MD, George D, Alsop DC. Arterial spin labeling blood flow magnetic resonance imaging for the characterization of 
metastatic renal cell carcinomal. Acad. Radiol. 2005;12:347-357. doi: 10.1016/j.acra.2004.12.012.

22. Schor-Bardach R, Alsop DC, Pedrosa I, et al. Does Arterial Spin-labeling MR Imaging-measured Tumor Perfusion Correlate with Renal Cell Cancer Response to Antiangiogenic Therapy in a Mouse Model? Radiology 2009;251:731-742. doi: 10.1148/radiol.2521081059.

23. Noll DC, Nishimura DG, Macovski A. Homodyne detection in magnetic resonance imaging. IEEE Trans. Med. Imaging 1991;10:154-163. doi: 10.1109/42.79473.

24. Dai W, Robson PM, Shankaranarayanan A, Alsop DC. Reduced resolution transit delay prescan for quantitative continuous arterial spin labeling perfusion imaging. Magn. Reson. Med. 2012;67:1252-1265. doi: 10.1002/mrm.23103.

25. Alsop DC, Detre JA. Reduced transit-time sensitivity in noninvasive magnetic resonance imaging of human cerebral blood flow. J. Cereb. Blood Flow Metab. Off. J. Int. Soc. Cereb. Blood Flow Metab. 1996;16:1236-1249. doi: 10.1097/00004647-19961100000019 .

26. Buxton RB, Frank LR, Wong EC, Siewert B, Warach S, Edelman RR. A general kinetic model for quantitative perfusion imaging with arterial spin labeling. Magn. Reson. Med. 1998;40:383-396.

27. Zhang X, Petersen ET, Ghariq E, De Vis JB, Webb AG, Teeuwisse WM, Hendrikse J, van Osch MJP. In vivo blood T(1) measurements at $1.5 \mathrm{~T}, 3 \mathrm{~T}$, and $7 \mathrm{~T}$. Magn. Reson. Med. 2013;70:1082-1086. doi: 10.1002/mrm.24550.

28. Garcia DM, Duhamel G, Alsop DC. Efficiency of inversion pulses for background suppressed arterial spin labeling. Magn. Reson. Med. 2005;54:366-372. doi: $10.1002 / \mathrm{mrm} .20556$.

29. Schraml C, Martirosian P, Schwenzer N., Boss A, Claussen C., Schick F. Functional perfusion imaging of the pancreas using an arterial spin labeling technique. In: Proc. Intl. Soc. Mag. Reson. Med. Toronto, ON, Canada; 2008. p. 2626.

30. Qiu M, Wang J, Pinus A, Kim H, Constable R. Arterial Spin Labeling TrueFISP Pancreas/Liver Perfusion Imaging at 1.5 Tesla. In: Proc. Intl. Soc. Mag. Reson. Med. Seattle, WA, USA; 2006. p. 3248.

31. Jansson L, Barbu A, Bodin B, et al. Pancreatic islet blood flow and its measurement. Ups. J. Med. Sci. 2016;121:81-95. doi: 10.3109/03009734.2016.1164769.

32. Wang X, Misawa R, Zielinski MC, et al. Regional Differences in Islet Distribution in the Human Pancreas - Preferential Beta-Cell Loss in the Head Region in Patients with Type 2 Diabetes. PLOS ONE 2013;8:e67454. doi: 10.1371/journal.pone.0067454. 
33. Dai W, Shankaranarayanan A, Alsop DC. Volumetric measurement of perfusion and arterial transit delay using hadamard encoded continuous arterial spin labeling. Magn. Reson. Med. 2013;69:1014-1022. doi: 10.1002/mrm.24335.

34. He X, Wengler K, Schweitzer ME. Diffusion sensitivity of 3D-GRASE in arterial spin labeling perfusion. Magn. Reson. Med. 2018;80:736-747. doi: 10.1002/mrm.27058.

35. Weigel M, Hennig J. Diffusion sensitivity of turbo spin echo sequences. Magn. Reson. Med. 2012;67:1528-1537. doi: 10.1002/mrm.24286. 


\section{Tables}

\begin{tabular}{|c|c|c|c|c|c|c|c|c|c|}
\hline Group & & & $(\mathbf{m}$ & & $\begin{array}{c}\text { Non-corrected } \\
\text { BF } \\
(\mathrm{mL} / 100 \mathrm{~g} / \mathrm{min})\end{array}$ & SNR & rSNRt & ATT (ms) & $\begin{array}{c}\text { Corrected BF } \\
(\mathrm{mL} / 100 \mathrm{~g} / \mathrm{min} \\
)\end{array}$ \\
\hline 1 & 1 & 816 & \pm & 50 & $168 \pm 37$ & 8.8 & 2.4 & \multirow{10}{*}{ N/A } & $140 \pm 30$ \\
\hline 1 & 2 & 859 & \pm & 97 & $255 \pm 64$ & 17.3 & 4.6 & & $217 \pm 52$ \\
\hline 1 & 3 & 815 & \pm & 121 & $214 \pm 64$ & 10.2 & 2.7 & & $177 \pm 49$ \\
\hline 1 & 4 & 914 & \pm & 67 & $150 \pm 33$ & 11.0 & 2.9 & & $130 \pm 29$ \\
\hline 1 & 5 & 740 & \pm & 120 & $271 \pm 103$ & 9.9 & 2.6 & & $217 \pm 77$ \\
\hline 1 & 6 & 1041 & \pm & 125 & $167 \pm 52$ & 8.6 & 2.3 & & $149 \pm 43$ \\
\hline 1 & 7 & 794 & \pm & 89 & $218 \pm 63$ & 8.6 & 2.3 & & $180 \pm 49$ \\
\hline 1 & 8 & 883 & \pm & 77 & $165 \pm 58$ & 8.9 & 2.4 & & $141 \pm 47$ \\
\hline 1 & 9 & 843 & \pm & 117 & $205 \pm 56$ & 16.7 & 4.5 & & $172 \pm 44$ \\
\hline 1 & 10 & 810 & \pm & 86 & $196 \pm 59$ & 13.9 & 3.7 & & $163 \pm 46$ \\
\hline Mean & & 852 & \pm & 95 & $201 \pm 59$ & 11.4 & 3.0 & \multirow{2}{*}{$\mathrm{N} / \mathrm{A}$} & $169 \pm 47$ \\
\hline$\pm \mathrm{SD}$ & & 82 & \pm & 26 & $40 \pm 19$ & 3.4 & 0.9 & & $31 \pm 13$ \\
\hline 2 & 11 & 719 & \pm & 89 & $213 \pm 61$ & \multirow{4}{*}{\multicolumn{2}{|c|}{$\mathrm{N} / \mathrm{A}$}} & $998 \pm 242$ & $177 \pm 61$ \\
\hline 2 & 12 & 902 & \pm & 244 & $183 \pm 80$ & & & $935 \pm 354$ & $165 \pm 71$ \\
\hline 2 & 13 & 892 & \pm & 191 & $185 \pm 68$ & & & $1147 \pm 239$ & $148 \pm 53$ \\
\hline 2 & 14 & 877 & \pm & 88 & $181 \pm 55$ & & & $1036 \pm 247$ & $158 \pm 55$ \\
\hline Mean & & 848 & \pm & 153 & $191 \pm 66$ & \multirow{2}{*}{\multicolumn{2}{|c|}{ N/A }} & $1029 \pm 271$ & $162 \pm 60$ \\
\hline \pm SD & & 86 & \pm & 78 & $15 \pm 11$ & & & $89 \pm 56$ & $12 \pm 8$ \\
\hline
\end{tabular}

Table 1 -Results from single and multi-delay ASL acquisitions 


\section{Figures}

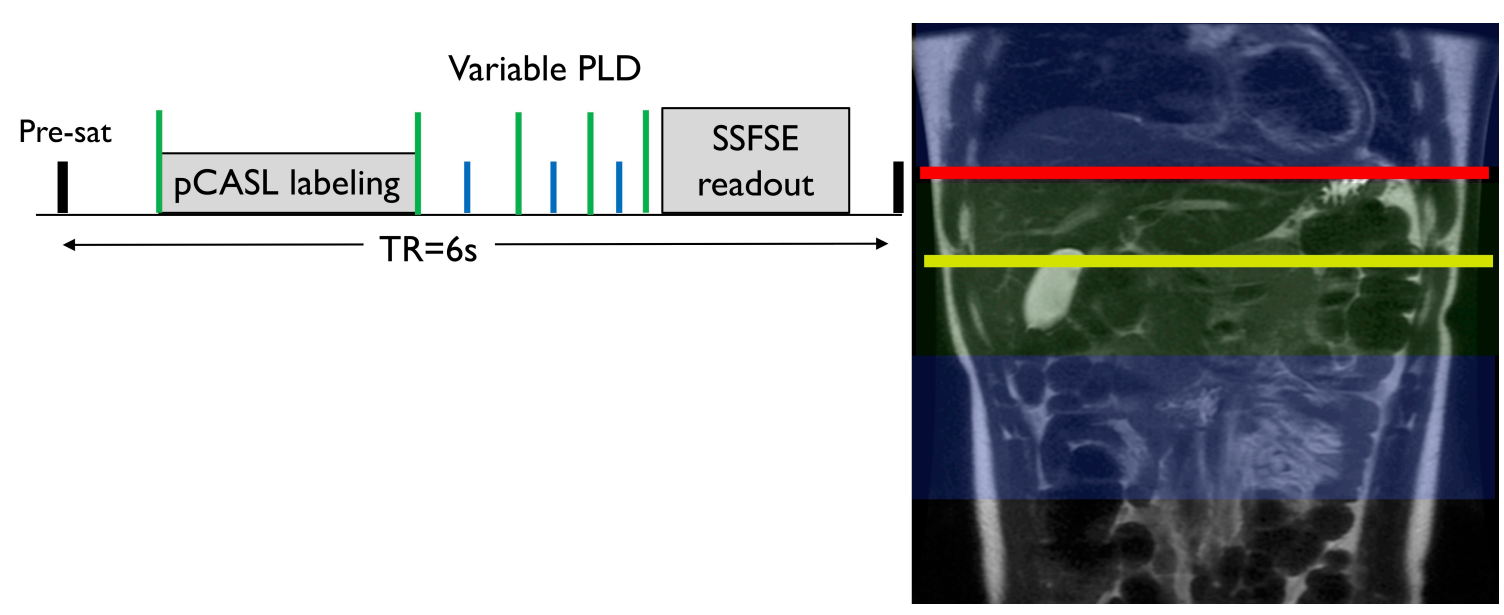

Figure 1 - Left - pCASL-SSFSE sequence timing. The green bars represent the various background suppression inversion pulses while the blue bars represent the different saturation pulses. Right - position of labeling plane (red), imaging slice (yellow) as well as BS extent (green overlay) superior/inferior saturation slabs (blue overlay) on a coronal $\mathrm{T}_{2}$ w SSFSE 

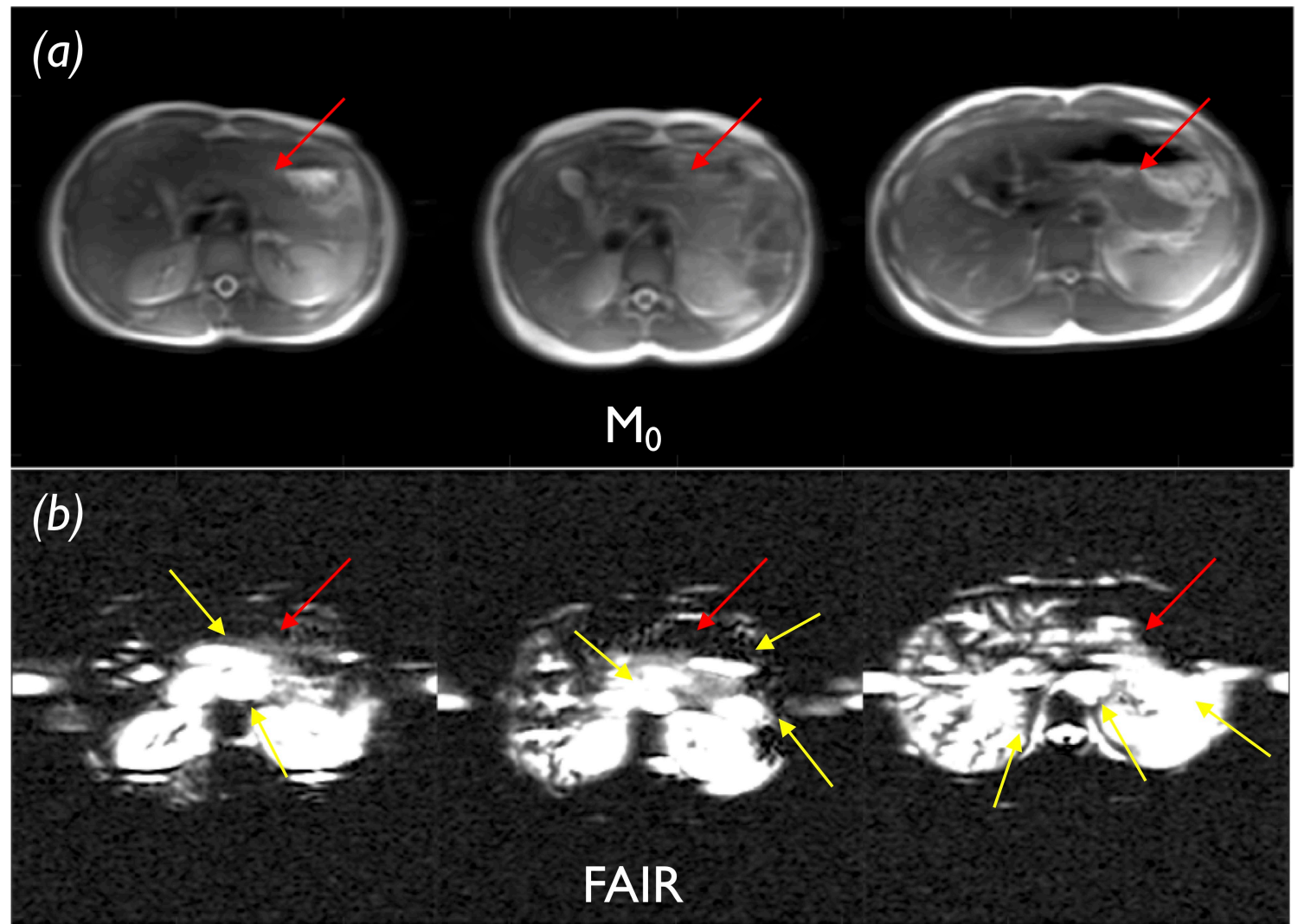

(c)

Figure $2-\mathrm{M}_{0}$ reference (top) and ASL perfusion difference images acquired with FAIR (middle) and pCASL (bottom) in 3 volunteers highlighting significant perfusion signal in the pancreas (red arrows). The yellow arrows on FAIR images highlight evidence of vascular contamination. Additionally, strong signal can also be seen in the spleen and kidneys 


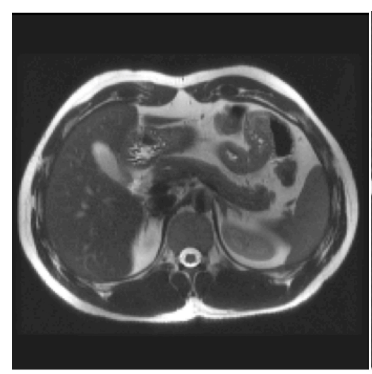

$T_{2}$-w SSFSE

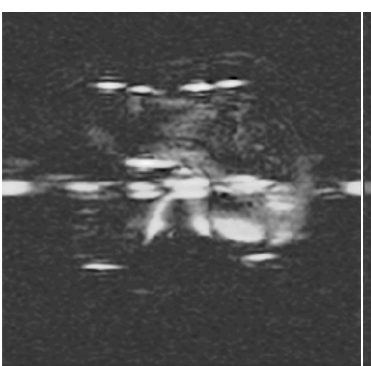

$0.7 \mathrm{~s}$

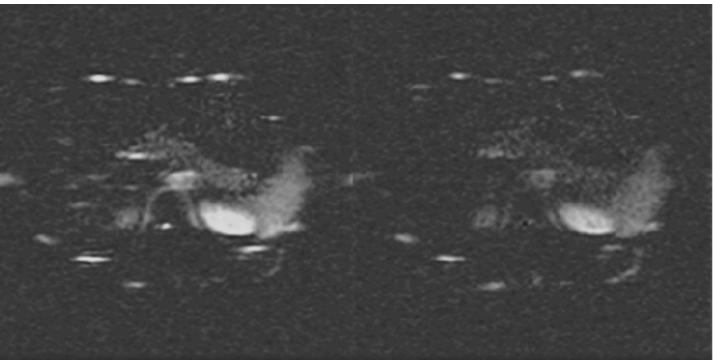

I.5s

$2 s$

ASL perfusion difference $(\mathrm{dM})$

PLD (s)

Figure 3 - Evolution of the ASL signal with various PLDs. 


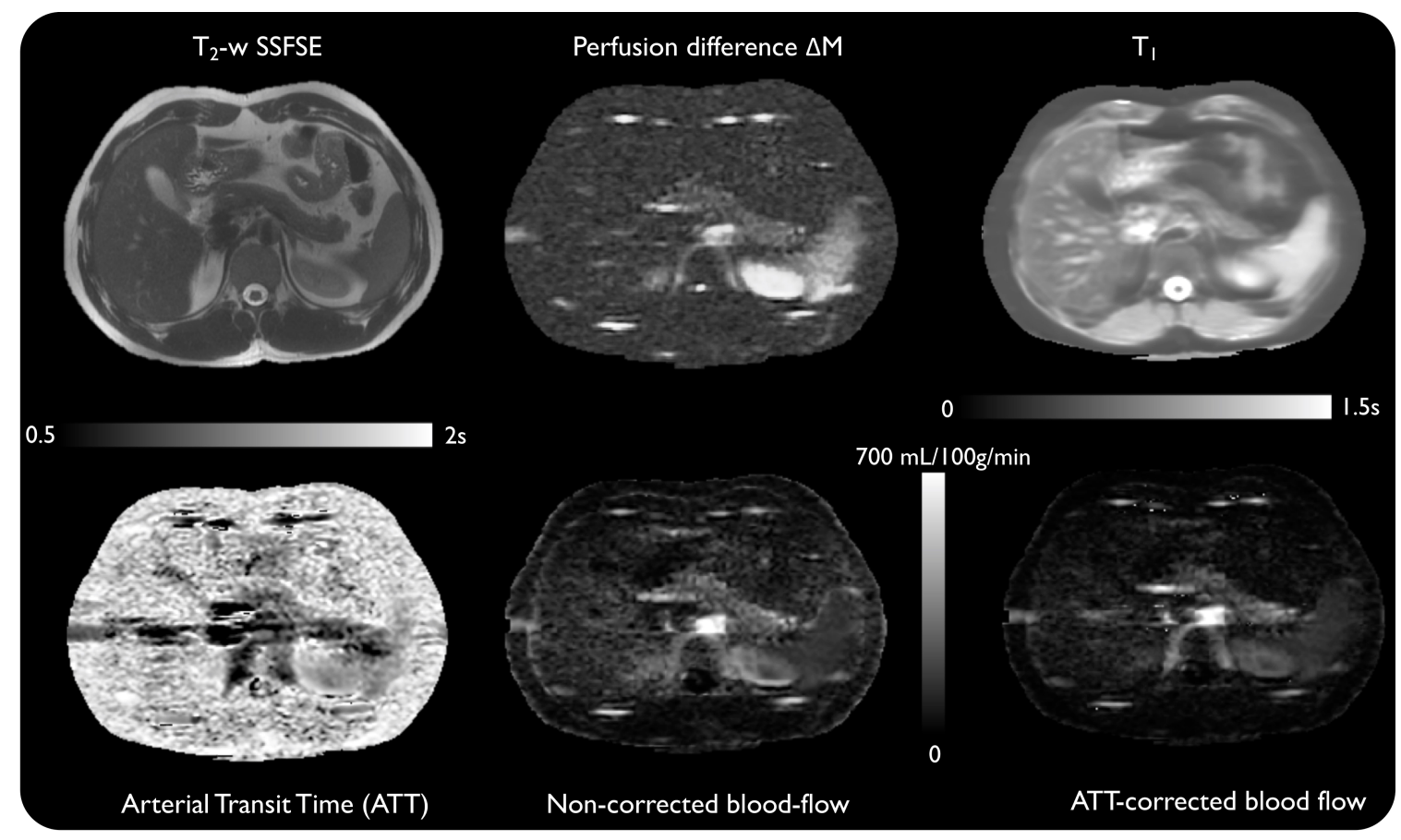

Figure 4 - Illustration of the influence of ATT correction on quantitative pancreatic bloodflow 

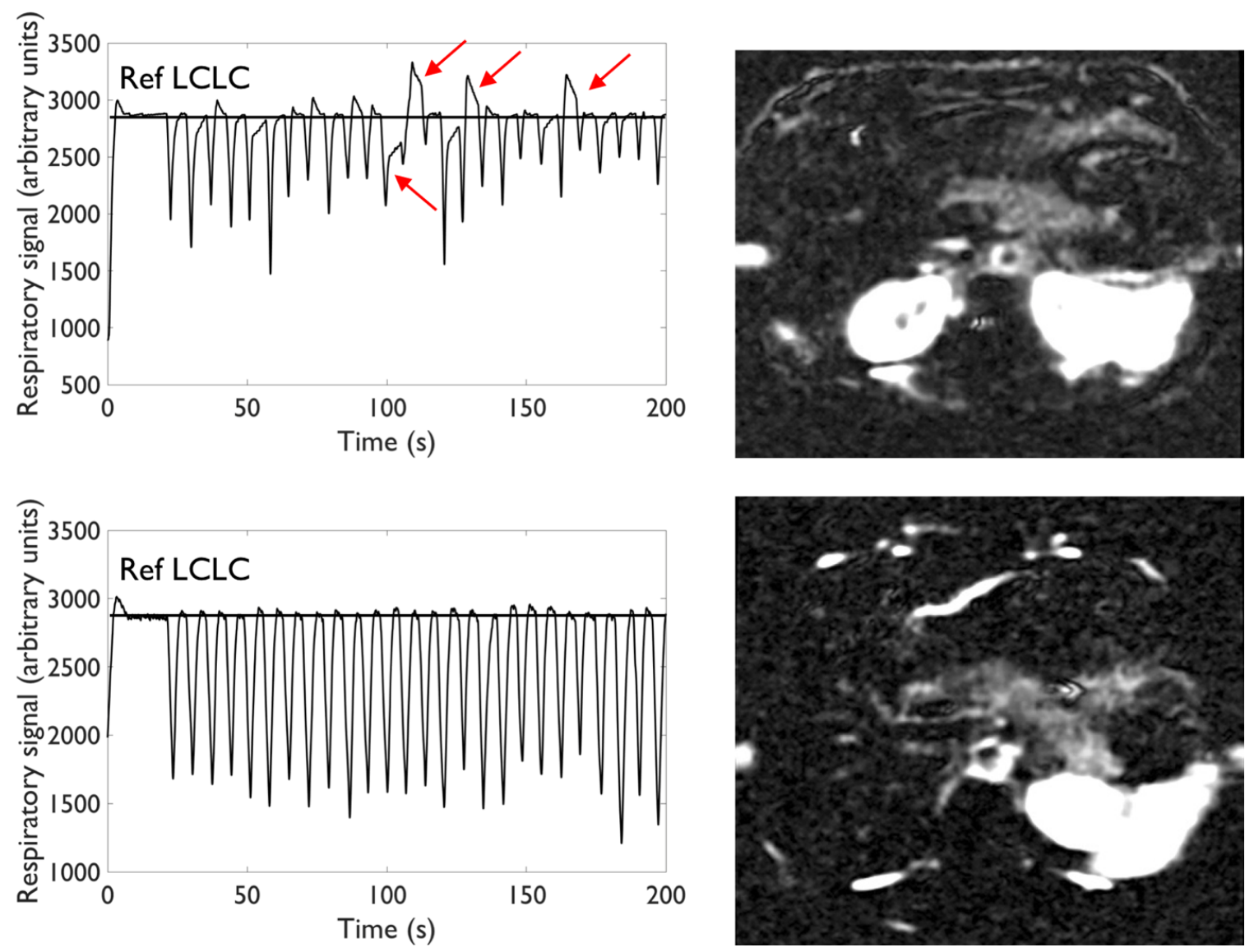

Supporting figure S1 - Respiratory trace (left) and resulting ASL perfusion difference image (right) in two cases with perfect compliance with breathing instructions (bottom) and some missed instructions (top, materialized by red arrows). Ref/L/C refers to the acquisition of reference, label and control images respectively 